UDC 316.4.066

LBC 60.561 .2

\title{
ACCOMMODATION FACILITIES AS THE MOST IMPORTANT FACTOR OF THE DEVELOPMENT OF THE TERRITORY'S TOURIST POTENTIAL
}

\author{
Evgeniy S. Vasiliev \\ Volgograd State University, Volgograd, Russian Federation \\ Elena V. Guryeva \\ Volgograd State University, Volgograd, Russian Federation
}

\begin{abstract}
The current stage of economic development of the Russian Federation is characterized by the search for new prospects and unrealized potentials, with the help of which it is possible not only to solve economic problems, but also to overcome the social difficulties that have developed over the past decades. The tourist industry is just such a promising area for further development. Possessing a unique combination of social and economic characteristics, the tourism industry places high demands on the conditions for planning and implementing strategic projects. One of the directions to improve the efficiency of the implementation of projects in the tourism sphere is the assessment of the potential in this activity field. This will improve strategic planning and control over the implementation of new projects. Widely used in economic sociology multi-criteria assessment methods are suitable tools for successful and correct determining the potential of various types of tourism. However, in the process of implementing this method at the stage of determining the weight coefficients, there is a high probability of error. Setting an incorrect weighting coefficient will negatively affect the final efficiency and reliability of the constructed socio-economic model, which will lead to incorrect initial data for conducting strategic analysis of the industry. In this study, in order to eliminate this problem, it is proposed to conduct a preliminary analysis to determine the importance of one of the elements of the tourism sector, namely accommodation facilities. Using existing statistical data and applying the method of correlation dependence the authors determine the influence of accommodation facilities in the tourism industry, as well as determine the basic spread of the weight coefficient for its further use in multi-criteria assessment methods of evaluation of socio-economic processes. As a result, it will allow to avoid the indicated danger and increase the reliability of determining the tourist potential of a particular type of tourism or tourist territory.
\end{abstract}

Key words: tourist potential, accommodation facilities, hotels, motels, hostels, multi-criteria analysis, cluster analysis, correlation dependence, weight coefficient.

УДК 316.4 .066

ББК 60.561 .2

СРЕДСТВА РАЗМЕЩЕНИЯ

КАК ФАКТОР РАЗВИТИЯ ТУРИСТСКОГО ПОТЕНЦИАЛА ТЕРРИТОРИИ

\author{
Евгений Сергеевич Васильев
}

Волгоградский государственный университет, г. Волгоград, Российская Федерация

Елена Владимировна Гурьева

Волгоградский государственный университет, г. Волгоград, Российская Федерация

Аннотация. Современный этап развития экономики Российской Федерации характеризуется поиском новых перспектив и нереализованных потенциалов, с помощью которых можно решить не только экономические задачи, но и преодолеть сложившиеся за последние десятилетия социальные трудности. Туристская индустрия как раз и является таким перспективным направлением дальнейшего развития. 
Обладая уникальным сочетанием социальных и экономических характеристик, сфера туризма предъявляет повышенные требования к условиям планирования и реализации стратегических проектов. Одним из направлений, которое позволит улучшить эффективность реализации проектов в сфере туризма, является оценка потенциала данной сферы деятельности. Что позволит осуществлять более качественное стратегическое планирование и контроль над реализацией новых проектов. Широко используемые в экономической социологии многокритериальные методы оценки являются подходящим инструментом для успешного и корректного определения потенциала различных видов туризма. Однако в процессе реализации данного метода на этапе определения весовых коэффициентов возникает высокая вероятность ошибки. Установление некорректного весового коэффициента отрицательно скажется на итоговой эффективности и достоверности сконструированной социально-экономической модели, что приведет к получению неверных начальных данных для проведения стратегического анализа отрасли. В данном исследовании для устранения указанной проблемы предлагается провести предварительный анализ, который позволит определить важность одного из элементов сферы туризма, а именно средств размещения. Используя существующие статистические данные, а также применяя метод корреляционной зависимости авторы определяют влияние средств размещения на развитие туристской индустрии, а также определяют базовый разброс весового коэффициента для дальнейшего его использования в многокритериальных методах оценки социально-экономических процессов. Что в итоге позволит избежать указанной опасности и повысит достоверность определения туристского потенциала конкретного вида туризма или туристской территории.

Ключевые слова: туристский потенциал, средства размещения, гостиницы, мотели, хостелы, многокритериальный анализ, кластерный анализ, корреляционная зависимость, весовой коэффициент.

\section{Введение}

Потребительское поведение акторов в сфере услуг на данный момент является одним из факторов социально-экономического развития современного государства. Данная тенденция связана с особенностью услуг, как «товара», который в силу его уникального «непостоянства» характеристик можно реализовывать практически бесконечное количество раз одному и тому же потребителю. Таким образом органично преодолевается функциональное ограничение, свойственное промышленной экономике.

Туризм представляет собой в большей степени отрасль оказания услуг, чем реализации товаров, является уникальным направлением экономической деятельности, обладающим практически неограниченным потенциалом воздействия на социальное, культурное, образовательное и экономическое развитие страны и на международные отношения. Туризм стимулирует экономическую деятельность за счет мультипликативного эффекта и развивается, используя местные культурные и природные особенности территорий. Уникальность туристской отрасли экономики проистекает из особенностей ее формирования, способности агрегировать множество достаточно самостоятельных видов деятельности, таких как услуги по организации питания, услуги транспорта, экскурсионные, рекреационные и спортивные услуги, а также услуги средств размещения.

Институциональная особенность формирования туристской отрасли оказывает существенное влияние и на методы ее исследования. Так, оценка потенциала проводится по видовому направлению туристской деятельности, что, с одной стороны, выглядит обоснованно, но с другой, обладает определенными недостатками, связанными с поверхностными оценками отдельных составляющих туристкой индустрии со стороны исследователей. В подобной ситуации становится важным определить место каждого составляющего элемента туристской индустрии при проведении оценки потенциала, сформировать и закрепить методы, наиболее качественно характеризующие текущее состояние отдельных составляющих элементов индустрии туризма.

\section{Методология}

«Туристический потенциал»- это широко используемый и общепринятый на международном уровне термин в сфере туризма, однако в некоторых случаях возможны определенные сложности с его употреблением, так как потенциал может выражать некоторые 
территориальные возможности, которые относятся к другим областям исследований. Поэтому Е. Богатовой и Т. Бочковой [Богатова, Бочкова 2017] термин «потенциал» предлагается заменить словом «привлекательность», что четко указывает на потребительское поведение и отношения между спросом и предложением туризма. Тем не менее, большая часть исследователей предпочитает использовать именно термин «потенциал», так как считают его более устоявшимся.

В настоящее время существуют многие практики оценки туристического потенциала, то есть количественной оценки всех аспектов или атрибутов для формирования одного интегрального показателя с использованием различных инструментов многокритериальных методов принятия решений. Данный метод получил широкое распространение среди зарубежных исследователей туристского потенциала. С. Иату и М. Булай предлагают метод множественной линейной регрессии для количественной оценки прибытия туристов на основе четырех переменных, а именно: природных ресурсов, культурных ресурсов, туристической инфраструктуры и общей инфраструктуры [Iatu, Bulai 2011]. И.-В. Сирие и др. предлагает метод масштабирования с весовыми значениями таких переменных, как природный потенциал, антропный потенциал, инфраструктура туризма и техническая инфраструктура [Ciurea et al. 2011].

Также широкое распространение получил и кластерный анализ, основанный на методе взвешенной линейной комбинации с применением анализа главных компонентов, он был предложен П. Ашури и Ш. Фариади [Ashouri, Fariyadi 2011]. Они взяли 15 переменных для оценки потенциала экотуризма. Используется кластерный анализ и российскими исследователями [Валеева 2017; Глоова 2011; Trukhachev 2015].

Еще один популярный способ оценки туристического потенциала и связанных с ним исследований - использование географической информационной системы (ГИС). Ф. Эффат и М.Н. Хегази предложили использовать многокритериальные методы в сочетании с картографическими данными, которые соответствующим образом накладываются друг на друга для получения рейтинга и взвешивания туристических достопримечательностей и инфраструктуры [Effat, Hegazy 2012]. Р. Махмудов и др. предлагают методологию, основанную на многослойных картах с систематическим анализом для определения туристического потенциала, а также направления дальнейшего развития в исследуемых районах [Maхмудов и др. 2016].

Особенностью использования многокритериальных методов принятия решений является необходимость применения весовых коэффициентов к каждому из используемых в методе критериев. Неверное решение на данному этапе может поставить под сомнение результативность любого из указанных выше методов. Для решения данной проблемы необходимо определить важность составляющих элементов туристкой индустрии в целом, провести анализ их развития и охарактеризовать степень их влияния на индустрию в целом. Определение такого «глобального» индекса заметно упростит принятие верного решения в процессе использования многокритериальных методов.

В рамках данного исследования мы постараемся провести оценку степени влияния средств размещения на формирование потенциала туристской индустрии. Необходимо дать конкретный ответ, как правильно оценивать вклад такой составляющей части туристской индустрии как средства размещения в развитие потенциала территорий.

\section{Результаты}

Для формирования отношения к средствам размещения как одному из составляющих многокритериальных методов оценки потенциала туристской индустрии воспользуемся данными статистических наблюдений, сформированных и представленных на сайте Федеральной службы государственной статистики [Федеральная служба государственной статистики...]. Инструментарий и методология, используемая для сбора официальной статистической отчетности, постоянно подвергаются заслуженной критике в связи с низкой детализацией и отсутствием показателей по ряду направлений деятельности в сфере туризма. Однако в нашем случае доступной информации будет достаточно для проведения 
анализа динамических рядов представленных показателей, а также поверхностного корреляционного анализа.

Обратимся к разделу показателей «Коллективные средства размещения». В первую очередь необходимо проанализировать динамику изменения состава средств размещения за последние 5 лет (см. табл. 1).

Анализ данных показывает, что за рассматриваемый период число средств размещения в целом выросло более чем в 2,5 раза. Необходимо отметить факт неоднородности данного роста по категориям внутри рассматриваемого показателя. Так гостиницы, составляющие в начале анализируемого периода $68 \%$ от общего числа средств размещения, росли наименее активно, всего на $112 \%$. Итогом послужило снижение их доли в общем числе средств размещения до $57 \%$ по данным 2019 года.

Наибольший рост демонстрирует развитие предложения среди хостелов, итоговый прирост за последние пять лет составил $225 \%$, т.е. число хостелов увеличилось в 3,25 раза. Однако нельзя не отметить, что данный бурный рост может свидетельствовать о социально-экономической нестабильности, что непосредственно влияет на потребительское поведение. В 2018 году число хостелов больше, чем в 2019 году, произошло заметное снижение их общего количества, падение составило 5,5\%.

Другие тенденции в сфере развития мотелей: в 2016 году хороший старт (+68 \% по отношению к 2015 году), к концу рассматриваемого периода их рост ограничивается $2 \%$, составив за весь период внушительные 159 \%, что больше, чем показатель роста числа гостиниц. Тем не менее, рост мотелей имеет по- ступательный характер, в отличие от роста хостелов.

Изменения, произошедшие за указанный период в структуре средств размещения, указывают на определенную стабильность. В 2020-2021 гг. не прогнозируется взрывного роста ни в одной из категорий средств размещения. Число мотелей и хостелов приблизилось к максимально возможному. Однако гостиницы имеют потенциал роста в размере 1$3 \%$ в течение еще 2-3 лет, если международный туризм не вернется на уровень 2019 г. в 2021 году. Заметные спонтанные флуктуации могут наблюдаться в сфере развития хостелов, так как затраты на их создание и закрытие минимальные по сравнению с другими категориями средств размещения, а, следовательно, ниже риски.

Для лучшего понимания распределения влияния на рынке между различными категориями средств размещения проанализируем объем оказанных услуг за аналогичный период (см. табл. 2).

Объем оказанных услуг, выраженный в количестве людей, размещенных в гостиницах и аналогичных средствах размещения, раскрывает новые особенности распределения среди категорий средств размещения. В течение всего анализируемого периода гостиницы, оставаясь основным поставщиком услуг по размещению, теряют доли внутри рынка. Только данная категория показала результат ниже среднего по рынку - 87 \% прироста при общем равном $100 \%$. Если в 2015 году 90 \% размещений обеспечивалось за счет гостиниц, то к концу рассматриваемого периода их доля составляет все еще внушительные, но 84 \%. Все потерянные проценты рынка достались хостелам, которые нара-

Таблииа 1

Состав и динамика средств размещения за период 2015-2019 гг., ед.

\begin{tabular}{|l|r|r|r|r|r|c|}
\hline \multicolumn{1}{|c|}{ Показатель } & 2015 & \multicolumn{1}{c|}{2016} & \multicolumn{1}{c|}{2017} & \multicolumn{1}{c|}{2018} & \multicolumn{1}{c|}{2019} & \multicolumn{2}{c|}{$2015 / 2019$} \\
\hline Число средств размещения & 8391 & 15368 & 18753 & 21302 & 21312 & $154 \%$ \\
\hline из них & & & & & & \\
\hline гостиницы & 5697 & 9513 & 10901 & 11676 & 12086 & $112 \%$ \\
\hline мотели & 167 & 281 & 381 & 422 & 432 & $159 \%$ \\
\hline хостелы & 535 & 928 & 1406 & 1833 & 1737 & $225 \%$ \\
\hline
\end{tabular}

Примечание. Источник: Составлено автором на основе данных Федеральной службы государственной статистики [Федеральная служба государственной статистики... гостиницы...]. 
стили долю своего присутствия с незначительных $2 \%$ в 2015 году до заметных 7 \% по итогам 2019 года. А если оценивать изменения объема услуг, оказанных за весь анализируемый период, то стоит констатировать невероятный рост в $628 \%$.

Тем не менее, стоит отметить, что в 2019 году происходит своего рода коррекция. Гостиницы возвращают $1 \%$ рынка за счет хостелов, что объясняется необоснованно высоким ростом их количества, за которым последовала естественная коррекция.

В целом объем предоставляемых услуг растет хорошими темпами (+100\%), однако данный рост отстает от роста количества самих средств размещения (напомним - это +154 \%). Для лучшего понимания ситуации рассмотрим эффективность использования своих возможностей всеми категориями средств размещения (см. табл. 3). Данный показатель позволит сделать окончательный вывод об основных трендах внутри самой категории.

Анализ данных, представленных в таблице 3 , позволяет с полной уверенностью зак- лючить, что основным драйвером рынка являются хостелы, нарастившие по итогам анализируемого периода свою эффективность на $124 \%$, и это при общей тенденции снижения эффективности среди остальных категорий средств размещения, так и в целом по рынку на $21 \%$.

Для лучшей наглядности выразим в графическом виде (см. рисунок). Дополнительно построенные линии трендов (пунктиры) подчеркивают описанный характер изменений.

Подведем итог анализу основных тенденций по динамике развития и изменениям в составе средств размещения за период 20152019 годов. Практически все тенденции положительны. Растет как количество средств размещения (+154\%), так и объем оказываемых ими услуг (+100 \%), однако при этом снижается эффективность использования (-21\%) собственных ресурсов. Структурные изменения в составе категорий средств размещения выявляют заметную тенденцию по перераспределению влияния в пользу хостелов. Данная категория средств размещения демонстрирует наиболее впечатляющие показатели

Таблииа 2

Объем оказанных услуг средствами размешения за период 2015-2019 гг., тыс. человек

\begin{tabular}{|l|r|r|r|r|r|c|}
\hline \multicolumn{1}{|c|}{ Показатель } & \multicolumn{1}{c|}{2015} & \multicolumn{1}{c|}{2016} & \multicolumn{1}{c|}{2017} & \multicolumn{1}{c|}{2018} & \multicolumn{1}{c|}{2019} & \multicolumn{1}{c|}{$2015 / 2019$} \\
\hline В средствах размещения & 30526 & 42981 & 48412 & 57243 & 61059 & \multicolumn{1}{c|}{$100 \%$} \\
\hline из них: & & & & & & \\
\hline в гостиницах & 27385 & 37549 & 42002 & 47291 & 51265 & \multicolumn{1}{c|}{$87 \%$} \\
\hline мотелях & 294 & 478 & 594 & 625 & 619 & $111 \%$ \\
\hline хостелах & 595 & 857 & 2147 & 4988 & 4333 & $628 \%$ \\
\hline
\end{tabular}

Примечание. Источник: Составлено автором на основе данных Федеральной службы государственной статистики [Федеральная служба государственной статистики... численность лиц...].

Загруженность средств размещения за период 2015-2019 гг., человек на 1 средство размещения

\begin{tabular}{|c|c|c|c|c|c|c|}
\hline \multicolumn{1}{|c|}{ Показатель } & \multicolumn{1}{c|}{2015} & \multicolumn{1}{c|}{2016} & \multicolumn{1}{c|}{2017} & \multicolumn{1}{c|}{2018} & \multicolumn{1}{c|}{2019} & $2015 / 2019$ \\
\hline В средствах размещения & 3637,9 & 2796,8 & 2581,5 & 2687,2 & 2865,0 & $-21 \%$ \\
\hline из них: & & & & & & \\
\hline в гостиницах & 4806,9 & 3947,1 & 3853,0 & 4050,3 & 4241,7 & $-12 \%$ \\
\hline мотелях & 1760,5 & 1700,1 & 1558,1 & 1482,0 & 1433,5 & $-19 \%$ \\
\hline хостелах & 1112,1 & 923,6 & 1526,8 & 2721,1 & 2494,6 & $124 \%$ \\
\hline
\end{tabular}

Примечание. Источник: Составлено автором на основе данных Федеральной службы государственной статистики: [Федеральная служба государственной статистики... гостиницы...; Федеральная служба государственной статистики... численность лиц...]. 
роста количества предложения, а также единственная из представленных в анализе категорий обеспечивает рост эффективности (+124\%) вопреки общему тренду.

Перейдем к анализу влияния индустрии средств размещения на сферу туризма (см. табл. 4).

Статистика по объему оказанных населению платных услуг в области туризма в целом положительна. По итогам анализируемого периода наблюдается рост в 23 \%. Если же рассмотреть состав формируемых услуг, то становится ясно, что основной вклад в данный объем вносят именно услуги средств размещения.

В течение всего рассматриваемого периода наблюдается тенденция увеличения доли услуг, оказываемых средствами размещения за счет снижения доли объема туристских услуг (см. табл. 5). Если в 2015 году доля услуг размещения в общем объеме оказанных услуг в сфере туризма составляла $54 \%$, то к 2019 году данная доля выросла до 58 \% (наблюдается небольшой «выброс» в 2018 году). Таким образом, объем оказанных платных услуг, оказанных средствами размещения за исследуемый период, вырос на $31 \%$, в то время как объем отдельных туристских услуг вырос лишь на 14 \%. Для выявления наличия зависимости между данными видами услуг рассчитаем корреляцию по методу Пирсона (для расчета берутся ряды данных в количестве 10 последних значений, предшествующих текущему году).

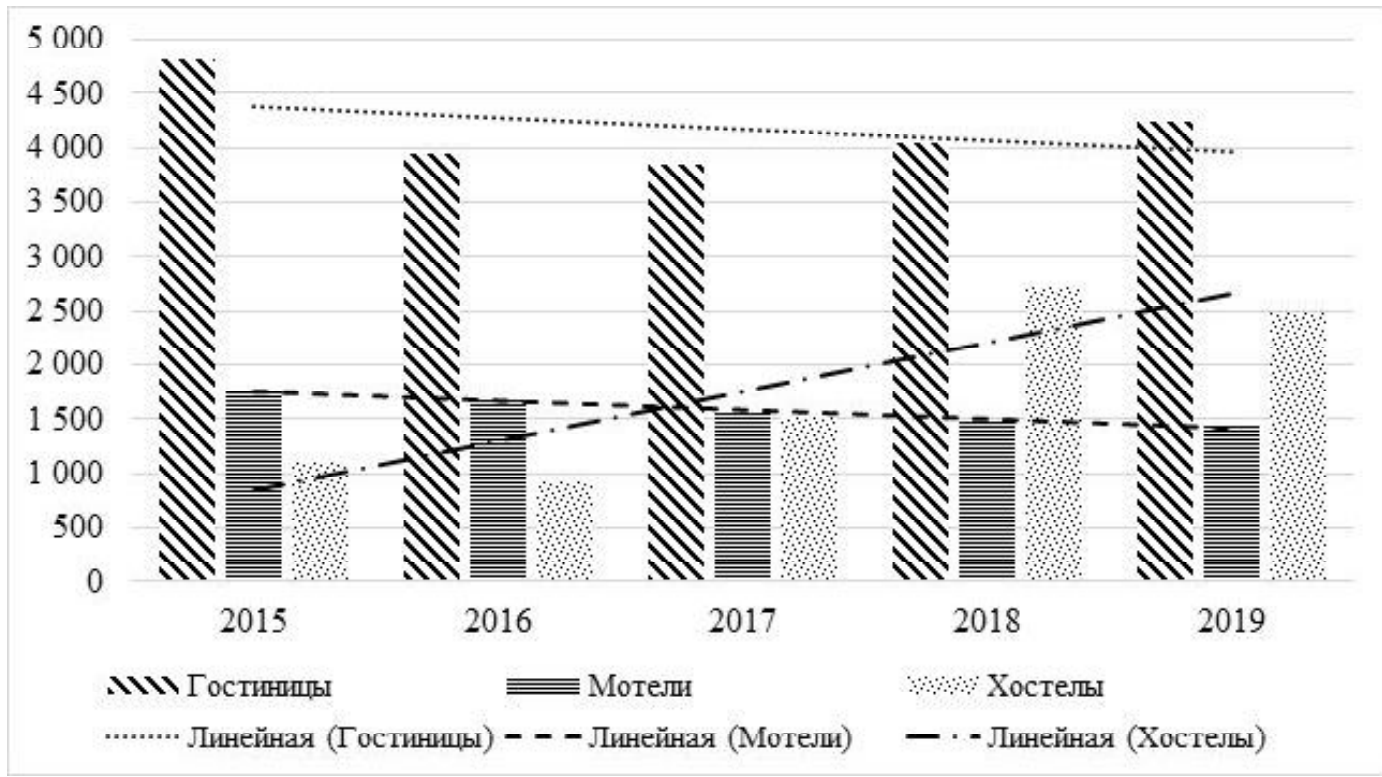

Загруженность средств размещения за период 2015-2019 гг., человек на 1 средство размещения

Примечание. Источник: Составлено автором на основе данных Федеральной службы государственной статистики: [Федеральная служба государственной статистики... гостиницы...; Федеральная служба государственной статистики... численность лиц...].

Таблииа 4

Объем платных услуг, оказанных в сфере туризма за период 2015-2019 гг., млн. руб.

\begin{tabular}{|l|c|c|c|c|c|c|}
\hline \multicolumn{1}{|c|}{ Показатель } & 2015 & 2016 & 2017 & 2018 & 2019 & $2015 / 2019$ \\
\hline Всего & 347244 & 374632 & 386436 & 427798 & 427105 & $23 \%$ \\
\hline из них: & & & & & & \\
\hline туристские услуги & 158252 & 161344 & 166520 & 172090 & 179826 & $14 \%$ \\
\hline услуги средств размещения & 188992 & 213288 & 219916 & 255708 & 247279 & $31 \%$ \\
\hline
\end{tabular}

Примечание. Источник: Составлено автором на основе данных Федеральной службы государственной статистики [Федеральная служба государственной статистики... Объем платных услуг...]. 
E.C. Васильев, E.В. Гурьева. Средства размещения как фактор развития туристского потенциала территории

Доля в формировании объема платных услуг, оказанных в сфере туризма

Таблица 5 за период 2015-2019 гг.

\begin{tabular}{|l|l|l|l|l|l|c|}
\hline \multicolumn{1}{|c|}{ Показатель } & 2015 & 2016 & 2017 & 2018 & 2019 & $2015 / 2019$ \\
\hline Туристские услуги & $46 \%$ & $43 \%$ & $43 \%$ & $40 \%$ & $42 \%$ & $-8 \%$ \\
\hline Услуги средств размещения & $54 \%$ & $57 \%$ & $57 \%$ & $60 \%$ & $58 \%$ & $6 \%$ \\
\hline Корреляция & 0,97 & 0,94 & 0,93 & 0,89 & 0,90 & - \\
\hline
\end{tabular}

Примечание. Источник: Составлено автором на основе данных Федеральной службы государственной статистики [Федеральная служба государственной статистики... Объем платных услуг...].

На протяжении всего анализируемого периода наблюдается снижение корреляции, то есть и зависимости объема оказываемых услуг средствами размещения и объема туристских услуг. Таким образом, можно сделать вывод о том, что за последние пять лет зависимость данных показателей друг от друга снизилась, однако она до сих пор остается довольно высокой. А степень влияния сферы средств размещения на туристскую индустрию можно охарактеризовать как прямо пропорциональную. Полученный вывод позволяет при использовании многокритериальных моделей определить разброс весового коэффициента, применяющийся к доле влияния сферы средств размещения на конкретную отрасль туристской индустрии, в пределах $0,5-0,6$ от суммы долей всего остального набора критериев, использующихся в конкретной модели.

\section{Выводы}

Проведенное исследование включало в себя два направления. Сначала была рассмотрена динамика изменения доступных показателей и состава средств размещения. Проведенный анализ позволяет охарактеризовать существующую динамику изменений как положительную: расчет число средств размещения (+154\%), увеличивается объем оказываемых ими услуг (+100 \%). Анализ состава средств размещения выявил важную тенденцию, связанную с активным развитием категории хостелов, повышении их роли (с 1 до 7 \%) в формировании показателей сфер средств размещения, а также очень заметный рост эффективности (+124\%) данной категории средств размещения по сравнению с более традиционными (гостиницы).
Наличие положительного заключения по характеру изменений в сфере средств размещения позволило перейти к следующему этапу анализа: выявлению степени и характера влияния сферы средств размещения на итоговые результаты работы многокритериальных моделей принятия решений или оценки потенциала какого-либо из видов туризма. Итоговое значение выявленного весового коэффициента составляет $0,5-0,6$ от суммы долей всего остального набора критериев, использующихся в конкретной модели.

Таким образом, полученные выводы показывают влияние сферы услуг размещения на индустрию туризма и характеризуют потребительское поведение акторов. Сфера размещения в течение последнего времени становится менее зависимой от индустрии туризма, о чем свидетельствует динамика коэффициента корреляции, что может быть следствием процесса замещения услуг. Тем не менее, эта тенденция может меняться в зависимости от социальноэкономических показателей территорий, и в ближайшие 3-5 лет можно прогнозировать баланс.

\section{СПИСОК ЛИТЕРАТУРЫ}

Богатова, Бочкова 2017 - Богатова Е.А., Бочкова T.A. Туризм в Краснодарском крае: система управления и потенциал // Теория и практика современной науки. 2017. № 3 (21). C. $116-120$.

Валеева 2017 - Валеева С.В. Кластер и технологическая платформа в управлении инновационным развитием туризма // Журнал правовых и экономических исследований. 2017. № 3. C. $180-182$.

Глоова 2011 - Глоова А.В. Кластерный подход как ключевой инструмент развития туризма в 
регионе // Экономика и экологический менеджмент. 2011. № 2. С. 26-33.

Махмудов и др. 2016 - Махмудов Р.К., Стоек Ю.О., Черкасов А.А. Проектирование и составление туристской карты Ставропольского края // Материалы Международной конференции «ИнтерКарто. ИнтерГИС». 2016. Т. 22 (2). C. $166-174$.

Федеральная служба государственной статистики... гостиницы... - Коллективныле средства размещзения, годовые данные, гостинииьы и аналогичные средства размещения // https:// rosstat.gov.ru/storage/mediabank/AvJTL8d1/51-9.xlsx (дата обращения: 05.12.2020).

Федеральная служба государственной статистики... численность лиц... - Коллективные средства размещения, годовые данные, численность лиц, размещуенных в гостиницах и аналогичных средствах размещения // https:// rosstat.gov.ru/storage/mediabank/A8PWVknJ/ 5-1-10.xlsx (дата обращения: 05.12.2020).

Федеральная служба государственной статистики... Объем платных услуг.. - Объем платных услуг населению // https://rosstat.gov.ru/storage/ mediabank/7-1.xlsx (дата обращения: 05.12.2020).

Ashouri, Fariyadi 2010-Ashouri P., Fariyadi Sh. Potential Assessment of Nature-Based Tourism Destinations Using MCA Techniques (Case Study: Lavasan-e Koochak) // Journal of Environmental Studies. 2010. Vol. 36, № 55. P. 1-12.

Ciurea et al. 2011 - Ciurea I.-V., Mihalache R., Ungureanu G., Brezuleanu S. Studies Regarding the Evaluation of the Tourist Potential of Oituz Hydrographical Basin - Bacau County // Bulletin UASVM Horticulture. 2011. Vol. 68, № 2. P. 49-54.

Effat, Hegazy 2012 - Effat H., Hegazy M.N. Mapping potential landfill sites for North Sinai cities using spatial multicriteria evaluation // The Egyptian Journal of Remote Sensing and Space Science. 2012. Vol. 15, № 2. P. 125-133.

Iatu, Bulai 2011 - Iatu C., Bulai M. New Approach in Evaluating Tourism Attractiveness in the Region of Moldavia (Romania) // International Journal of Energy and Environment. 2011. Vol. 5. P. $165-174$.

Trukhachev 2015 - Trukhachev A. Methodology for Evaluating the Rural Tourism Potentials: A Tool to Ensure Sustainable Development of Rural Settlements // Sustainability. 2015. № 7. P. 3052-3070.

\section{REFERENCES}

Bogatova E.A., Bochkova T.A., 2017. Tourism in the Krasnodar Territory: management system and potential. Theory and practice of modern science, no. 3 (21), pp. 116-120.

Valeeva S.V., 2017. Cluster and technology platform in the management of innovative tourism development. Journal of Legal and Economic Studies, no. 3, pp. 180-182.

Gloova A.V., 2011. Cluster Approach as a Key Tool for Tourism Development in the Region. Economics and environmental management, no. 2, pp. 26-33.

Mahmudov R.K., Stoek J.O., Cherkasov A.A., 2016. Designing and composition of tourist maps of Stavropol region. Proceedings of the International conference «InterCarto. InterGIS», vol. 22 (2), pp. 166-174.

Federal State Statistics Service... Hotels... Collective Accommodation Facilities, Annual Data, Hotels and Similar Accommodation Facilities. URL: https://rosstat.gov.ru/storage/mediabank/ AvJTL8d1/5-1-9.xlsx (accessed 05 December 2020).

Federal State Statistics Service... Number of Persons... Collective Accommodation Facilities, Annual Data, Number of Persons Accommodated in Hotels and Similar Accommodation Facilities. URL: https://rosstat.gov.ru/storage/mediabank/ A8PWVknJ/5-1-10.xlsx (accessed 05 December 2020).

Federal State Statistics Service... The Volume of Paid Services... The Volume of Paid Services to the Population. URL: https://rosstat.gov.ru/ storage/mediabank/7-1.xlsx (accessed 05 December 2020).

Ashouri P., Fariyadi Sh., 2010. Potential Assessment of Nature-Based Tourism Destinations Using MCA Techniques (Case Study: Lavasan-e Koochak). Journal of Environmental Studies, vol. 36, no. 55, pp. 1-12.

Ciurea I-V., Mihalache R., Ungureanu G., Brezuleanu S., 2011. Studies Regarding the Evaluation of the Tourist Potential of Oituz Hydrographical Basin - Bacau County. Bulletin UASVM Horticulture, vol. 68, no. 2, pp. 49-54.

Effat H., Hegazy M.N., 2012. Mapping Potential Landfill Sites for North Sinai Cities Using Spatial Multicriteria Evaluation. The Egyptian Journal of Remote Sensing and Space Science, vol. 15, no. 2, pp. 125-133.

Iatu C., Bulai M., 2011. New Approach in Evaluating Tourism Attractiveness in the Region of Moldavia (Romania). International Journal of Energy and Environment, vol. 5, pp. 165-174.

Trukhachev A., 2015. Methodology for Evaluating the Rural Tourism Potentials: A Tool to Ensure Sustainable Development of Rural Settlements. Sustainability, no. 7, pp. 3052-3070. 
E.C. Васильев, E.В. Гурьева. Средства размещения как фактор развития туристского потенциала территории

\section{Information About the Authors}

Evgeniy S. Vasiliev, Candidate of Sciences (Economics), Head of the Innovation Activities Office, Volgograd State University, Prosp. Universitetsky, 100, 400062 Volgograd, Russian Federation, vasileves@volsu.ru, https://orcid.org/0000-0002-8798-9880

Elena V. Guryeva, Senior Lecturer, Department of Service and Tourism, Volgograd State University, Prosp. Universitetsky, 100, 400062 Volgograd, Russian Federation, elena.guryeva@volsu.ru, https:// orcid.org/0000-0002-4870-7609

\section{Информация об авторах}

Евгений Сергеевич Васильев, кандидат экономических наук, заведующий отделом инновационной деятельности, Волгоградский государственный университет, просп. Университетский, 100, 400062, г. Волгоград, Российская Федерация, vasileves@volsu.ru, https://orcid.org/ 0000-0002-8798-9880

Елена Владимировна Гурьева, старший преподаватель кафедры сервиса и туризма, Волгоградский государственный университет, просп. Университетский, 100, 400062, г. Волгоград, Российская Федерация, elena.guryeva@volsu.ru, https://orcid.org/0000-0002-4870-7609 